\title{
Probing collective dynamics of active particles using modulation force spectroscopy
}

\author{
G. V. Soni \\ National Center for Biological Sciences, Tata Institute of Fundamental Research, Bangalore 560065, India \\ G. Ananthakrishna \\ Materials Research Center \& Center for Condensed Matter Theory, Indian Institute of Science, Bangalore \\ 560012, India \\ G. V. Shivashankar ${ }^{a)}$ \\ National Center for Biological Sciences, Tata Institute of Fundamental Research, Bangalore 560065, India \\ and Raman Research Institute, CV Raman Avenue, Bangalore 560080, India
}

\begin{abstract}
In this letter, we report a method of measuring the dynamic viscosity of self-propelled active particles using an intensity-modulated optical tweezer. We have used a $6 \mu \mathrm{m}$ trapped polystyrene bead suspended in a bath of motile bacterial cells as a probe. The response function amplitude of the oscillatory bead directly measures the dynamics of the spatiotemporal structure of the motile particles. We find that unlike passive systems, the viscosity is defined by distributions of response function amplitudes that represent the long-range active temporal structures. Appropriate Langevin equations are set up that capture all these essential features.
\end{abstract}

Pattern formation of self-propelled active particles in a random media is of interest in biological systems and in the context of correlated dynamics in soft matter. ${ }^{1-3}$ In a recent report, we studied the onset of dynamic correlation in selfpropelled system of motile bacterial cells using an optical tweezer. ${ }^{4}$ These experiments used a dc optical tweezer and single particle tracking methods to analyze the time series of the Brownian fluctuations of the trapped bead with and without bacteria. In the absence of an appropriate theoretical framework for the dc experiments, we could only extract an effective dynamic viscosity arising from the nonequilibrium nature of active bacterial bath. However, as the active particles are known to form spatially coherent structures with a finite lifetime, the dc optical tweezer experiments were inadequate to capture the dynamical correlations arising from these structures of the active bacteria. In this letter, we present a method of using an intensity-modulated (ac) optical tweezer to probe the temporal dynamics of the bacterial aggregates in the bath. Here, the response function amplitude $(R)$ corresponding to the frequency of the modulated optical tweezer probes the lifetimes of the coherent structures in the local fluid bath. Thus, the probability distribution of the response function amplitude $P(R)$ is used to differentiate and characterize the active and passive rheology, not accessible through a dc optical tweezer. Langevin equations appropriate to the situation are set up that capture all the essential features observed in experiments.

Schematic of the experimental setup is shown in Fig. 1(a). The optical tweezer was set up by focusing an infrared laser beam (SDL-5400 series diode, $830 \mathrm{~nm}, 200 \mathrm{~mW}$ SDL Inc. CA, USA, with L-3724B laser diode controller from ILX Lightwave Corporation, Montana, USA) to a diffraction-limited focus using an objective lens (100X, N.A

\footnotetext{
a) Author to whom correspondence should be addressed; electronic mail: shiva@ncbs.res.in
}

1.4, Olympus, Japan). A red diode laser $(5 \mathrm{~mW}, 635 \mathrm{~nm}$, Thorlabs, Newton, NJ) was aligned coaxial to the infrared laser for tracking the bead in the trap using backscattered light collected on a quadrant detector. ${ }^{5}$ The laser power was modulated at a predetermined frequency $\omega_{0}$ by the internal sinewave generator of the lock-in amplifier (LIA) (model SR830 DSP, Stanford Research Systems, CA). The time series of the trapped bead fluctuations in the intensitymodulated trap was obtained, with and without bacterial bath, using onboard DAQ (data acquisition card PCI-MIO -16EX10) and analysis was carried out using Labview software (National Instruments, Austin, TX). The QD amplifier output was given as input to the LIA and the frequency of trap modulation as the reference frequency. The lock-in amplifier gives the imaginary $V_{i}$ and real $V_{r}$ components of the response of the bead fluctuation at the modulating signal frequency from which the amplitude $\left(R=\left(V_{r}^{2}+V_{i}^{2}\right)^{1 / 2}\right)$ and the phase $\left(\phi=\tan ^{-1}\left(V_{i} / V_{r}\right)\right)$ of the bead response are calculated. One hundred points were collected in $20 \mathrm{~s}$ from the LIA (with an averaging time of $0.2 \mathrm{~s}$ ), which is adequate for sampling the dynamics, but is short enough to ensure that there is no significant sedimentation of the bacteria in the medium. The sample was stirred and then left for homogenization for about $30 \mathrm{~s}$ between any two experiments. Each experiment lasted for $2 \mathrm{~h}$ from the time of resuspension of the cells in the motility buffer. The motility buffer does not favor bacterial growth and thus the cell number remains unchanged. We have also confirmed, by microscopy, that the cell shape and size is not changed during the experiment.

Figure 1(b) shows plots of the histograms of the response function amplitude $(R)$ for various viscosities corresponding to different glycerol concentrations. Comparing the curves for water and glycerol, it is clear that for a given frequency, $R$ is viscosity dependent. In water, $R$ has larger mean and higher dispersion compared to that at higher viscosities for which the peak is located close to zero. The mean value of $R$ in water $\left(\eta_{\text {water }}=1\right)$ is 0.14 and in glycerol $70 \%$ 

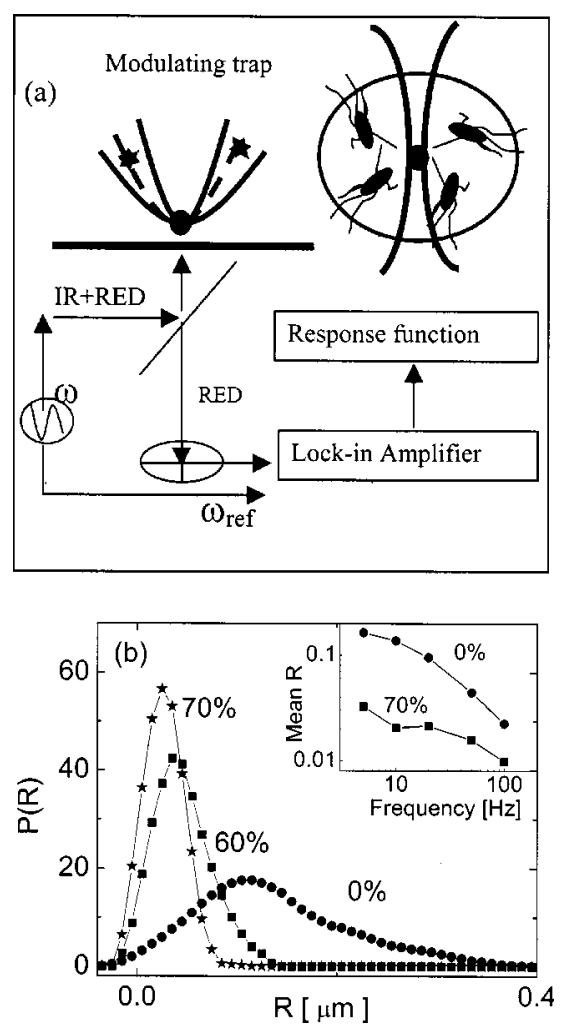

FIG. 1. (a) Schematic of the experiment. (b) Histograms of the response function amplitude of a bead trapped at different passive viscosities (at different glycerol concentrations) at trap modulation frequency $\omega_{0}=10 \mathrm{~Hz}$. Inset: The mean $R$ values for $0 \%$ and $70 \%$ glycerol concentration as a function of frequency.

$\left(\eta_{\text {glycerol }}=23\right)$ is 0.02 and thus the response function amplitude is a good measure of these changes. In Fig. 1(b) (inset), we plot the mean response function amplitude $(R)$ as a function of the trap modulation frequency. In general for any given viscosity, as expected, mean $R$ decreases with increasing frequency. We note that the time scales of the forcing frequency is long enough to allow the bead to relax completely in the trap potential.

In experiments on bacteria, ${ }^{6}$ we have used the time scales of measurement of $R$ to be $\sim 20 \mathrm{~s}$ intervals, which is much smaller than the sedimentation time scales. In this context, it is pertinent to note that in the absence of sedimentation, one should expect to probe all allowed configurations in the stationary state by monitoring the system over long time scales instead of the $20 \mathrm{~s}$ periods. However, as sedimentation is unavoidable, in each run lasting $20 \mathrm{~s}$ one expects to sample different coherent configurations present due to the active bacterial dynamics during this period. In order to show the effect of correlated bacterial dynamics, a comparative plot of the distributions of $R$ for motile (active) bacteria, dead (passive) bacteria, along with water $\left(\eta_{\text {water }}=1\right)$ and glycerol $\left(\eta_{\text {glycerol }}=23\right)$ is presented in Fig. 2 . It is clear that runs 1 and 2 are very different. Further, from the trend seen in Fig. 1(b) namely, the shift in the peak of the distribution of $R$ to lower values for higher viscosity, one can conclude that these two responses should correspond to two distinct configurations of the active bacterial dynamics. This also shows that different structures of the coherent bacteria lead to different viscosities in different runs, showing variability in the structure density, dynamics, and interaction with the probe particle. Note that the concentration of the active bacteria

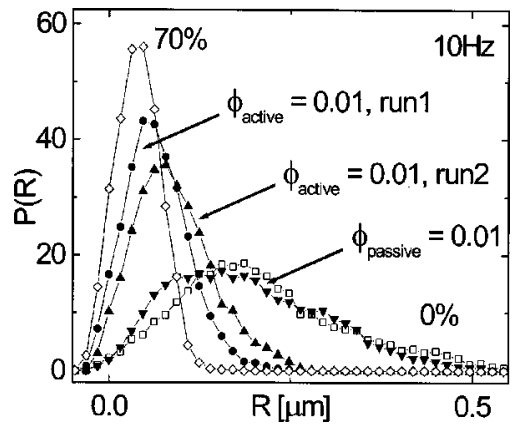

FIG. 2. Probability distribution of response amplitude of the trapped bead at $10 \mathrm{~Hz}$ laser intensity modulation in(a) 70\% glycerol, (b) $0 \%$ glycerol, (c) active and passive bacterial bath is plotted. Two representative experimental runs for $\phi_{\text {active }}=0.01$ are shown.

was chosen such that the dynamic viscosity arising due to their presence is similar to that of $70 \%$ glycerol. Interestingly, for the same concentration of passive bacteria, we find that the behavior of $R$ is similar to that of water implying the absence of aggregation.

In order to understand these results, we first model the power spectrum of the trapped bead in a bacterial bath without modulation. ${ }^{4}$ The motion of the bead trapped in a harmonic potential embedded in a viscous medium is usually described by ignoring the inertial effects using an overdamped Langevin equation:

$$
\gamma^{d x} / d t=-k x+\xi_{1}(t)+F_{B} .
$$

Here $x$ is the displacement of the bead in the trap, $\gamma$ is the viscosity term given by $\gamma=6 \pi \eta r$ ( $r$ is the bead radius and $\eta$ the bare viscosity), $k$ is the trap stiffness, $\xi_{1}(t)$ is the thermal white noise and $F_{B}$ is the average external force imparted by the bacterial clusters on the bead. The bacterial clusters of varying sizes are subject to viscous damping and thermal fluctuations described by

$$
F_{B}=m^{d v} /_{d t}=-\beta v+\xi_{2}(t),
$$

where $m$ is the mass of the bacterial cluster (assumed to be composed of a few bacteria), $\beta$ is the damping constant related to the bacterial cluster viscosity, i.e., $\beta=6 \pi \eta R$ ( $R$ is the cluster size), and $\xi_{2}(t)$ is the thermal white noise as experienced by the bacterial cluster. The statistical properties of noise satisfy

$$
\begin{aligned}
& \left\langle\xi_{i}(t)\right\rangle=0, \\
& \left\langle\xi_{i}(t) \xi_{j}\left(t^{\prime}\right)\right\rangle=2 \alpha_{i} \delta_{i j} k_{B} T \delta\left(t-t^{\prime}\right),
\end{aligned}
$$

where $i, j=1,2$ and $\alpha_{1}=\gamma$ and $\alpha_{2}=\beta$. Using $F_{B}=-\beta v$ in Eq. (1), and going through a simple calculation gives the power spectral density of $x$,

$$
S_{\beta v}(\omega)=\frac{2 \gamma k_{B} T}{\gamma^{2} \omega^{2}+k^{2}}\left[1+\frac{\beta^{3}}{\gamma} \frac{1}{\omega^{2} m^{2}+\beta^{2}}\right] .
$$

We note here that the effect of the bacteria enters through the mass factor or $\beta$. Thus, for $m=0$, (or $\beta=0$ ), we recover the power spectrum density of the bead in the absence of the bacteria, i.e., $\phi=0$. Further, we note that the effect of bacterial concentration is reflected in the zero frequency limit of the power spectral density for any given $\phi$ to that at $\phi=0$ given by $S_{\beta v}(\phi, \omega=0) / S_{\beta v}(\phi=0, \omega=0)=1+\beta / \gamma$. This relation can be used to get an estimate of the mean size of the 

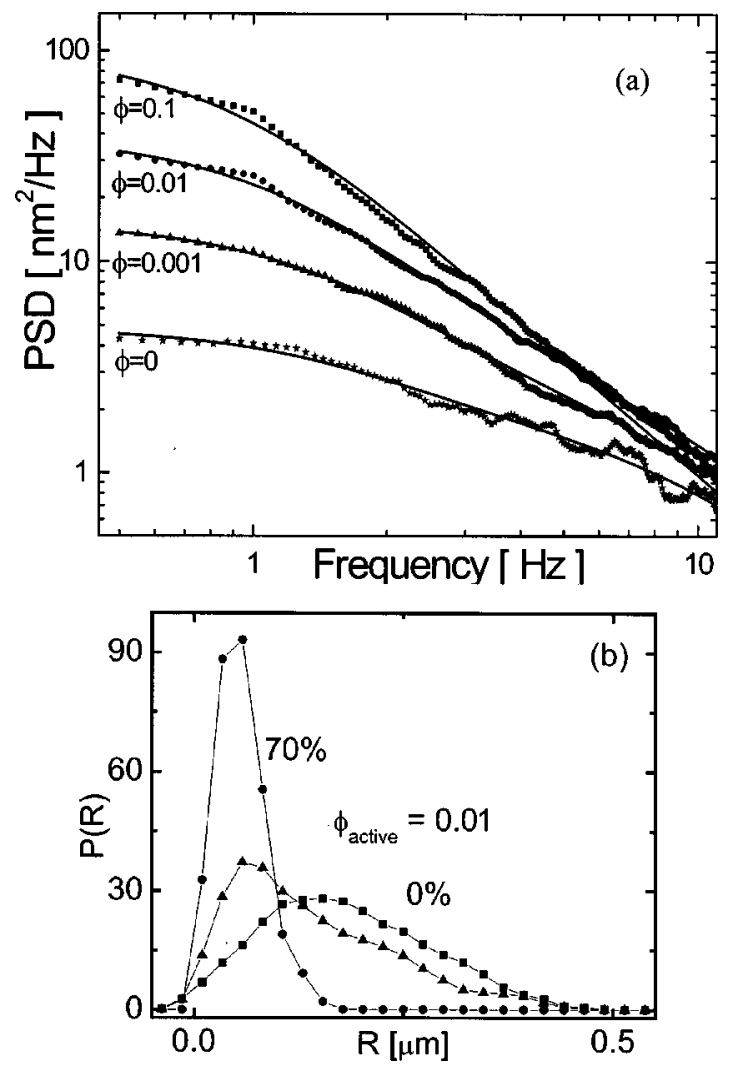

FIG. 3. (a) The power spectrum density for the fluctuation time series of a particle in a dc trap $\left(\omega_{0}=0 \mathrm{~Hz}\right)$ with different volume fractions of bacteria (Ref. 4). The solid lines are a fit to Eq. (4). (b) The simulation result of the ac trap experiment (nonzero $\omega_{0}$ ) using the model Eqs. (1), (2), and (5).

clusters. Assuming $\beta=a \gamma$, where $\beta$ is the hydrodynamic scaling of size of the clusters formed at any given $\phi$, we get $a=2.1,6.4$, and 15.7 for $\phi=0.001,0.01$, and 0.1 , respectively. Thus the probe encounters on an average fifteen bacterium at the largest experimental concentration in a coherent structure (need not be in a spherical form). It is known from previous work $^{2}$ that the bacterial motility follows a Maxwellian velocity distribution with an average velocity of $20 \mu \mathrm{m} / \mathrm{s}$. Using this it would require about $2.25 \mathrm{~s}$ for a string of 15 bacteria to cross a 3- $\mu \mathrm{m}$-diam confocal volume, an estimate that appears reasonable. Further, as shown in Fig. 3(a), Eq. (4) fits the power spectrum density data for volume fractions $\phi=0.1,0.01$, and 0.001 very well.

For the case of frequency-dependent modulation, we use the stiffness constant to have the form

$$
k=k_{0}+\delta k \sin (\omega t) \text {. }
$$

We have carried out numerical simulations of the Langevin dynamics. The parameters used in the simulation are $k_{0}$ $=2.36 \times 10^{-6} \mathrm{~N} / \mathrm{m}, \delta k=1.38 \times 10^{-6} \mathrm{~N} / \mathrm{m}$, and $\omega_{0}=10 \mathrm{~Hz}$. The width of the initial position is fixed to be within $50 \mathrm{~nm}$, consistent with the experiments. The time series generated from simulations are processed exactly as in experiments, i.e., the output time series of the simulation go through a virtual LIA and we extract the response function amplitude at the given frequency $\omega_{0}$. The averaging is carried out over a comparable number of histories (30). The distributions of the amplitude $R$ are plotted for different experimental conditions namely, for glycerol-70\%, bacteria $(\phi=0.01)$, and for water. As shown in Fig. 3(b), the distributions obtained from simulations are qualitatively similar to those seen in experiments [Fig. 2(b)] including the range and positions of the maxima.

In summary, we have developed an approach of using an intensity-modulated optical tweezer to probe the distribution in the effective dynamic viscosity arising due to long-range ordering of bacterial cells. The study shows that the variability in the Fourier amplitude of the bead displacement at the driving frequency $\left(\omega_{0}\right), R$, is a good measure of the local rheological properties of the fluid bath. Bacterial cells dynamically form multiple temporal structures of short lifetimes $(\sim 1 \mathrm{~s})$ and our probe directly captures the presence of these short-lived bacterial structures. Using the Langevin approach, we have captured all the dominant features of the long-range active correlations in the motile bacterial bath. The study demonstrates that fluctuations of a particle trapped in an ac trap provide an improved method of measuring frequency-dependent phenomena at increased signal to noise ratio. Such an approach has applications in probing local microrheological properties using a Brownian particle embedded in a suspension of diverse viscous solutions. ${ }^{7-10}$

The authors thank Professor J. S. Parkinson, University of Utah, Salt Lake City, for providing us with the bacterial strains used in this work.

${ }^{1}$ H. C. Berg, Proc. Natl. Acad. Sci. U.S.A. 93, 14225 (2000).

${ }^{2}$ X. L. Wu and A. Libchaber, Phys. Rev. Lett. 84, 3017 (2000).

${ }^{3}$ R. A. Simha and S. Ramaswamy, Phys. Rev. Lett. 89, 058101 (2002).

${ }^{4}$ G. V. Soni, B. M. Jaffar Ali, Y. Hatwalne, and G. V. Shivashankar, Biophys. J. 84, 2634 (2003).

${ }^{5}$ G. V. Soni, F. Meeran Hameed, T. Roopa, and G. V. Shivashankar, Curr. Sci. 83, 1464 (2002).

${ }^{6}$ Cells (E. Coli bacteria RP437) taken from plates were grown in $120 \mathrm{ml}$ $\mathrm{LB}$ media for $18 \mathrm{~h}$ at $25^{\circ} \mathrm{C}$. Cells were visually checked for their motile behavior, centrifuged at $5500 \mathrm{rpm}$ for $20 \mathrm{~min}$ at $4{ }^{\circ} \mathrm{C}$ and resuspended in the motility buffer (MB, $10 \mathrm{mM} \mathrm{KH} 2 \mathrm{PO} 4,67 \mathrm{mM} \mathrm{NaCl}, 0.1 \mathrm{mM}$ EDTA and $0.1 \%$ glycerol $)$ to the required concentration $\left(\sim 10^{8}\right.$ cells $/ \mathrm{ml}, \phi$ $=0.01)$. Cells were incubated at room temperature in the motility buffer for $30 \mathrm{~min}$ before the start of experiments. Measurements were carried out at room temperature using a $6 \mu \mathrm{m}$ trapped bead to track the bacterial dynamics. Sample cell volume was confined to $120 \mu \mathrm{l}$ in a circular well of depth $1.5 \mathrm{~mm}$ made of neoprene O-rings between two cover slips.

${ }^{7}$ M. L. Gardel, M. T. Valentine, J. C. Crocker, A. R. Bausch, and D. A. Weitz, Phys. Rev. Lett. 91, 158302 (2003).

${ }^{8}$ T. Roopa, N. Kumar, S. Bhattacharya, and G. V. Shivashankar, Biophys. J. 87, 974 (2004).

${ }^{9}$ N. B. Viana, R. T. S. Freire, and O. N. Mesquita, Phys. Rev. E 65, 041921 (2002).

${ }^{10}$ Y. Hatwalne, S. Ramaswamy, M. Rao and R. A. Simha, Phys. Rev. Lett. 92, 118101 (2004). 